\title{
Deeply virtual meson production on neutrons
}

\author{
William Brooks, Ivan Schmidt, and Marat Siddikov \\ Departamento de Física, Universidad Técnica Federico Santa María, \\ y Centro Científico-Tecnológico de Valparaíso, Casilla 110-V, Valparaíso, Chile
}

(Received 24 October 2018; published 18 December 2018)

\begin{abstract}
In this paper we analyze in detail how the measurements of exclusive electroproduction of mesons on neutrons would complement the studies of generalized parton distributions (GPDs) of the proton, providing independent experimental observables. Some of these processes on neutrons have very distinctive features, and thus we expect that measurements on liquid deuterium would allow to clearly distinguish them from similar processes on protons, giving a very clean probe of the GPD. In the case of charged meson production, all produced hadrons are charged, and for this reason we expect that the kinematics of this process could be easily reconstructed. We estimate the cross sections in the kinematics of the Jefferson Laboratory experiments using current phenomenological GPD models.
\end{abstract}

DOI: $10.1103 /$ PhysRevD.98.116006

\section{INTRODUCTION}

Understanding the structure of the hadrons nowadays is one of the major goals of high energy physics, and therefore it occupies a central place in the program of modern accelerator facilities. Today, this structure is parametrized in terms of the so-called generalized parton distributions (GPDs) which can be studied in Bjorken kinematics in a wide class of processes [1,2]. While the number of processes which can be used for studies of GPDs is rather large [3-11], the precision analyses are currently performed with deeply virtual Compton scattering (DVCS) [12-19] and deeply virtual production of light mesons (DVMP) [20-43], with most of the existing studies focusing on proton (liquid hydrogen) targets or production off protons inside nuclei. There are fewer studies of exclusive processes on neutrons [44-48], which might be partially due to the technical difficulties with accessing them experimentally, or perhaps the belief that they probe "the same" GPDs as in the case of protons. Given the fact that the amplitude of the hard exclusive process gets contributions from up to a dozen different GPDs, and that a large number of additional assumptions are involved in their modeling, we believe that studies on neutron are well justified, since neutron-induced processes provide independent observables and can also help to constrain the GPDs of the proton. For the sake of definiteness, in this paper we will focus on the deeply virtual production of pions and kaons

Published by the American Physical Society under the terms of the Creative Commons Attribution 4.0 International license. Further distribution of this work must maintain attribution to the author(s) and the published article's title, journal citation, and DOI. Funded by SCOAP ${ }^{3}$. on neutrons, tacitly implying that such measurements might be done on liquid deuterium $\left(\mathrm{LD}_{2}\right)$ with minimal uncertainty from nuclear effects. The feasibility of such measurements (although at small energies and virtualities too low for consideration in the Bjorken limit) was recently demonstrated experimentally in [45-48], and with the higher energies now available, it is feasible to make measurements in the Bjorken regime. The deeply virtual meson production is quite challenging because, as was found experimentally, the cross section of this process is dominated by contributions from poorly known transversity GPDs [49-51] convoluted with poorly known twist-3 distributions of mesons, and under the additional assumption that the three-parton distributions are negligible. While the processes on neutrons also require the use of model assumptions, we believe that they provide independent observables which might allow to test the GPDs extracted from analysis of DVMP on protons. Some of the processes on neutrons have distinctive features, and for this reason their measurement on liquid deuterium potentially provides a very clean channel for study of GPDs. As will be explained in the next section, the neutron GPDs either allow to probe new flavor combinations, or when contribute to the same combinations as on the proton, have better sensitivity to the region of negative light-cone fractions $x$, usually attributed to sea quarks. We also comment briefly on possible studies of the strange mesons $\left(K^{+}, K_{L, S}^{0}\right)$ production processes, which together with strangeness production on protons [51-55], might allow for a better understanding of the valence $u$ - and $d$-quarks GPDs: the $S U(3)$ relations [56] allow to relate the nucleonhyperon transition GPDs to the quark GPDs of the proton, and the cross sections of these processes do not get contributions from gluon GPDs nor from sea quarks 
(provided sea quarks are flavor symmetric, as assumed in most parametrizations of the GPDs).

The paper is organized as follows. In Sec. II we describe the framework used for the evaluation, and explain in detail the advantages of neutron-induced processes. The leading twist contributions are discussed in Sec. II A, and in Sec. II B we review the corrections due to transversity GPDs. Finally, in Sec. III we present numerical results using currently available models of GPDs, and draw conclusions.

\section{CROSS SECTION OF THE DVMP PROCESS}

As was demonstrated in $[3,14,37,57]$, the cross section for the deeply virtual exclusive meson production, $\gamma^{*}(q) N\left(p_{1}\right) \rightarrow N^{\prime}\left(p_{2}\right) M$ might be written in the form

$$
\begin{aligned}
2 \pi \frac{d \sigma}{d t d \varphi}= & {\left[\epsilon \frac{d \sigma_{L}}{d t}+\frac{d \sigma_{T}}{d t}+\sqrt{\epsilon(1+\epsilon)} \cos \varphi \frac{d \sigma_{L T}}{d t}\right.} \\
& +\epsilon \cos (2 \varphi) \frac{d \sigma_{T T}}{d t}+\sqrt{\epsilon(1+\epsilon)} \sin \varphi \frac{d \sigma_{L^{\prime} T}}{d t} \\
& \left.+\epsilon \sin (2 \varphi) \frac{d \sigma_{T^{\prime} T}}{d t}\right]
\end{aligned}
$$

where $\varphi$ is the angle between the lepton scattering and meson production planes, $t=\left(p_{1}-p_{2}\right)^{2}$ is the invariant momentum transfer. The cross sections also depend on the virtuality $Q^{2}=-q^{2}$ of the intermediate photon, where $q$ is its momentum, and the Bjorken variable $x_{B}=Q^{2} / 2 p_{1} \cdot q$. We also used standard shorthand notations,

$$
\epsilon=\frac{1-y-\frac{\gamma^{2} y^{2}}{4}}{1-y+\frac{y^{2}}{2}+\frac{\gamma^{2} y^{2}}{4}},
$$

for the ratio of transverse and longitudinal photon fluxes, where

$$
\gamma=\frac{2 m_{N} x_{B}}{Q}, \quad y=\frac{Q^{2}}{s_{e p} x_{B}}=\frac{Q^{2}}{2 m_{N} E_{e} x_{B}} .
$$

The cross section of the subprocess $\gamma^{*} n \rightarrow M n^{\prime}$ is related to the partial amplitudes as $[55,57]$

$$
\begin{gathered}
\frac{d \sigma_{L}}{d t}=\Gamma \sigma_{00} \\
\frac{d \sigma_{T}}{d t}=\Gamma\left(\frac{\sigma_{++}+\sigma_{--}}{2}+r_{L} \sqrt{1-\epsilon^{2}} \frac{\sigma_{++}-\sigma_{--}}{2}\right) \\
\frac{d \sigma_{L T}}{d t}=-\Gamma\left(\operatorname{Re}\left(\sigma_{0+}-\sigma_{0-}\right)+r_{L} \sqrt{\frac{1-\epsilon}{1+\epsilon}} \operatorname{Re}\left(\sigma_{0+}+\sigma_{0-}\right)\right) \\
\frac{d \sigma_{T T}}{d t}=-\Gamma \operatorname{Re}\left(\sigma_{+-}\right)
\end{gathered}
$$

$$
\begin{gathered}
\frac{d \sigma_{L^{\prime} T}}{d t}=\Gamma\left(\operatorname{Im}\left(\sigma_{+0}+\sigma_{-0}\right)+r_{L} \sqrt{\frac{1-\epsilon}{1+\epsilon}} \operatorname{Im}\left(\sigma_{-0}-\sigma_{+0}\right)\right), \\
\frac{d \sigma_{T^{\prime} T}}{d t}=\Gamma \operatorname{Im}\left(\sigma_{+-}\right) \\
\Gamma=\frac{1}{32 \pi\left(W^{2}-m^{2}\right) \Lambda\left(W^{2},-Q^{2}, m^{2}\right)}
\end{gathered}
$$

where $r_{L}$ is the polarization of the lepton beam, $\Lambda$ stands for the Mandelstam function $\Lambda(x, y, z)=$ $\sqrt{x^{2}+y^{2}+z^{2}-2 x y-2 x z-2 y z}$, and the subindices $\alpha$, $\beta$ of the matrix $\sigma$ refer to the polarization states of the intermediate photon in the amplitude and its conjugate. The matrix $\sigma_{\alpha \beta}$ is related to the helicity amplitudes $\mathcal{A}_{\nu^{\prime} 0, \nu \beta}$, by

$$
\sigma_{\alpha \beta}=\sum_{\nu \nu^{\prime}} \mathcal{A}_{\nu^{\prime} 0, \nu \alpha}^{*} \mathcal{A}_{\nu^{\prime} 0, \nu \beta}
$$

where $\nu, \nu^{\prime}$ are the polarization subindices of the initial and final hadrons. The amplitudes $\mathcal{A}$ carry all the information about the structure of the hadron. It is expected that in the formal Bjorken limit $\left(Q^{2} \rightarrow \infty, x_{\mathrm{B}}=\right.$ const) the dominant contribution comes from the leading twist term, $\sigma_{00}$, whereas all the other contributions should be suppressed at least as $\sim \mathcal{O}\left(m_{N} / Q\right)$. However, as was found in [51], in the JLab kinematics we are far from this regime, and the contributions of other harmonics in certain channels might yield contributions on par with the leading twist result. In the following subsections (II A, II B) we discuss in detail the contributions for the leading and subleading twists and the information on GPDs which they carry. In what follows we will consider only the case of unpolarized beams $\left(r_{L}=0\right)$ and targets, since the corresponding asymmetries might be observed only if the target is polarized, and in the case of neutrons inside a nucleus this presents a difficult technical problem [58-61] and requires modeling of nuclear interactions [62]. In this limit, the cross sections $d \sigma_{L^{\prime} T}(7)$ and $d \sigma_{T^{\prime} T}(8)$ vanish.

\section{A. Leading twist contribution}

The amplitude of the physical process in the formal Bjorken limit $\left(Q^{2} \rightarrow \infty\right)$ factorizes into convolution of the hard and the soft parts, as shown in Fig. 1:

$\mathcal{A}_{\nu^{\prime}, \nu}(\xi, t)=\int_{-1}^{+1} d x \sum_{q} \mathcal{H}_{\nu^{\prime} \lambda^{\prime}, \nu \lambda}^{q}\left(x, \xi, t, \mu_{F}\right) \mathcal{C}_{\lambda \lambda^{\prime}}^{q}\left(x, \xi, \mu_{F}\right)$,

where the sum runs over all parton flavors; $\lambda, \lambda^{\prime}$ are helicities of partons, $\nu, \nu^{\prime}$ are the helicities of the initial and final hadron, the skewness $\xi$ is related to light-cone 

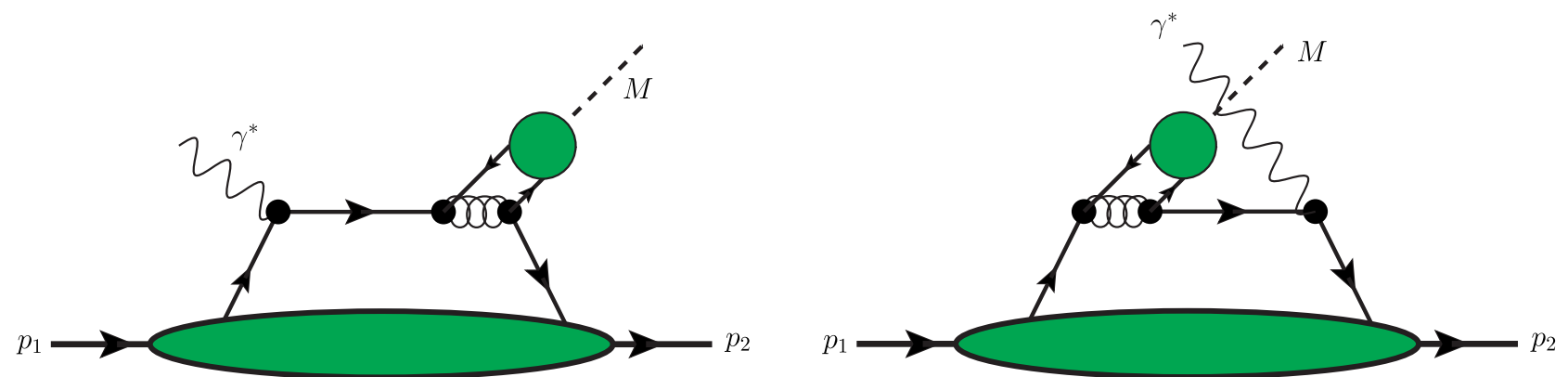

FIG. 1. Leading-order contributions to the DVMP hard coefficient functions, $s$-channel (left) and $u$-channel (right). The horizontal green blob stands for the generalized parton distributions of the parton, and the upper (small) green blob is the wave function of the produced meson. In the next-to-leading order, we should add additional gluon in all possible ways to the upper part of the diagram. The two-parton twist-3 contributions have the same structure.

momenta of the proton $p_{1,2}$ before and after interaction as $\xi=\left(p_{1}^{+}-p_{2}^{+}\right) /\left(p_{1}^{+}+p_{2}^{+}\right) \approx x_{B} /\left(2-x_{B}\right), \mu_{F}$ is the factorization scale, and all the other variables were defined earlier (see e.g., [3,27] for details of the kinematics). The soft matrix elements $\mathcal{H}^{q}$ in (11) are diagonal in quark helicities $\left(\lambda, \lambda^{\prime}\right)$ at leading twist, and can be parametrized in terms of four quark GPDs $H, E, \tilde{H}$, $\tilde{E}$ as

$\mathcal{H}_{\nu^{\prime} \lambda^{\prime}, \nu \lambda}^{q}=\frac{2 \delta_{\lambda \lambda^{\prime}}}{\sqrt{1-\xi^{2}}}\left(-\left(\begin{array}{cc}\left(1-\xi^{2}\right) H^{q}-\xi^{2} E^{q} & \frac{\left(\Delta_{1}+i \Delta_{2}\right) E^{q}}{2 m} \\ -\frac{\left(\Delta_{1}-i \Delta_{2}\right) E^{q}}{2 m} & \left(1-\xi^{2}\right) H^{q}-\xi^{2} E^{q}\end{array}\right)_{\nu^{\prime} \nu}+\operatorname{sgn}(\lambda)\left(\begin{array}{cc}-\left(1-\xi^{2}\right) \tilde{H}^{q}+\xi^{2} \tilde{E}^{q} & \frac{\left(\Delta_{1}+i \Delta_{2}\right) \tilde{\xi}^{q}}{2 m} \\ \frac{\left(\Delta_{1}-i \Delta_{2}\right) \tilde{E}^{q}}{2 m} & \left(1-\xi^{2}\right) \tilde{H}^{q}-\xi^{2} \tilde{E}^{q}\end{array}\right){ }_{\nu^{\prime} \nu}\right)$.

For the processes in which the baryon state changes, e.g., $e n \rightarrow e \pi^{-} p$, the transition GPDs are linearly related via the $S U(3)$ relations [56] to ordinary GPDs. The socalled coefficient functions $\mathcal{C}^{q}$ in (11) are the parton-level amplitudes and are evaluable in perturbative QCD. They might be represented as a sum of the $s$ - and $u$-channel contributions,

$$
\mathcal{C}^{q}(x, \xi)=\mathcal{C}_{s \text {-channel }}^{q}(x, \xi)+\mathcal{C}_{u \text {-channel }}^{q}(x, \xi),
$$

as shown in Fig. 1. In the Bjorken limit, these functions have an extremely simple form,

$\left.\begin{array}{l}\mathcal{C}_{s-\text { channel }}^{q(\text { LO })} \\ \mathcal{C}_{u-\text { channel }}^{q(\text { LO })}\end{array}\right\}=\eta_{q}^{(\mp)} c_{\mp}^{(q)}(x, \xi)+\mathcal{O}\left(\frac{m^{2}}{Q^{2}}\right)+\mathcal{O}\left(\alpha_{s}^{2}\left(\mu_{R}^{2}\right)\right)$,

where $\eta_{q}^{(\mp)}$ are some process-dependent flavor factors, and we introduced a shorthand notation,

$$
\begin{aligned}
c_{ \pm}^{(q)}(x, \xi)= & \left(\int d z \frac{\phi_{2, M}(z)}{z}\right) \frac{8 \pi i}{9} \frac{\alpha_{s}\left(\mu_{R}^{2}\right) f_{M}}{Q} \\
& \times \frac{1}{x \pm \xi \mp i 0}\left(1+\frac{\alpha_{s}\left(\mu_{r}^{2}\right)}{2 \pi} T^{(1)}\left(\frac{\xi \pm x}{2 \xi}, z\right)\right),
\end{aligned}
$$

where $\phi_{2, M}(z)$ is the twist-2 distribution amplitude (DA) of the produced meson [63]; the next-to-leading order (NLO) correction $T^{(1)}$ was evaluated in [33,64-67] and for the sake of completeness is given explicitly in the Appendix. While the coefficient function is known up to NLO $[33,64-$ 67], currently there is no detailed NLO GPDs (especially taking into account strange quarks) available from the literature. For this reason we will stick to the LO expressions. From the structure of (15) we may see that the $s$ channel amplitude probes the GPDs near the point $x \approx \xi \approx x_{B} /\left(2-x_{B}\right)$, a region dominated by the valence quarks. At the same time, after trivial algebraic rewrite,

$\int_{-1}^{+1} d x \mathcal{H}^{(q)}(x, \xi) c_{+}^{(q)}(x, \xi)=-\int_{-1}^{+1} d x \mathcal{H}^{(q)}(-x, \xi) c_{-}^{(q)}(x, \xi)$,

we observe that the $u$-channel amplitude might be interpreted as a probe of the GPDs at negative $x \approx-\xi$, the region 
TABLE I. Values of the flavor factors $\eta_{q}^{( \pm)}$for $q=u, d, s$ quarks. $e_{q}=\left\{\frac{2}{3},-\frac{1}{3},-\frac{1}{3}\right\}$ are the electric charges of the quarks. For the sake of reference in the right column we also placed the corresponding processes on a proton target which were previously studied in the literature.

\begin{tabular}{lcccc}
\hline \hline Process & $\eta_{+}^{q}$ & $\eta_{-}^{q}$ & Process & $\eta_{+}^{q}$ \\
\hline$e n \rightarrow e \pi^{-} p$ & $e_{u}\left(\delta_{q u}-\delta_{q d}\right)$ & $e_{d}\left(\delta_{q u}-\delta_{q d}\right)$ & $e p \rightarrow e \pi^{+} n$ & $e_{d}\left(\delta_{q u}-\delta_{q d}\right)$ \\
$e n \rightarrow e \pi^{0} n$ & $\frac{e_{u} \delta_{q d}-e_{d} \delta_{q u}}{\sqrt{2}}$ & $\frac{e_{u} \delta_{q d}-e_{d} \delta_{q u}}{\sqrt{2}}$ & $e p \rightarrow e \pi^{0} p$ & $\eta_{u}^{q}$ \\
$e n \rightarrow e K^{0} \Lambda$ & $-e_{s} \frac{2 \delta_{q d}-\delta_{q u}-\delta_{q s}}{\sqrt{6}}$ & $-e_{d} \frac{2 \delta_{q d}-\delta_{q u}-\delta_{q s}}{\sqrt{6}}$ & $e p \rightarrow e K^{+} \Lambda$ & $\left.-\delta_{q d}\right)$ \\
$e n \rightarrow e K^{0} \Sigma_{0}$ & $-e_{s} \frac{\delta_{q u}-\delta_{q s}}{\sqrt{2}}$ & $-e_{d} \frac{\delta_{q u}-\delta_{q u}}{\sqrt{2}}$ & $e p \rightarrow e \delta^{+} \delta_{0 d}-\delta_{q s}$ & $-e_{u} \delta_{q u}-e_{d} \delta_{q d}$ \\
$\sqrt{2}$ & $-e_{u} \frac{2 \delta_{q u}-\delta_{q d}-\delta_{q s}}{\sqrt{6}}$ & $e_{s} \frac{\delta_{q d}-\delta_{q s}}{\sqrt{2}}$ & $-e_{s}\left(\delta_{q d}-\delta_{q s}\right)$ \\
$e n \rightarrow e K^{+} \Sigma^{-}$ & $-e_{s}\left(\delta_{q u}-\delta_{q s}\right)$ & $-e_{u}\left(\delta_{q u}-\delta_{q s}\right)$ & $e p \rightarrow e K^{0} \Sigma^{+}$ & $-e_{d}\left(\delta_{q d}-\delta_{q s}\right)$ \\
\hline \hline
\end{tabular}

dominated by sea quarks. The values of the flavor factors are summarized in Table I and determine the sensitivity of each process to valence and sea quark GPDs.

For example, the charged pion $\left(\pi^{+}\right)$production on a proton is sensitive only to the combination of GPDs,

$\mathcal{H}_{p \rightarrow \pi^{+} n}\left(x, \xi, t, \mu_{F}\right) \equiv \frac{2}{3} \mathcal{H}^{(3)}\left(x, \xi, t, \mu_{F}\right)+\frac{1}{3} \mathcal{H}^{(3)}\left(-x, \xi, t, \mu_{F}\right)$,

where $\mathcal{H}^{(3)}\left(x, \xi, t, \mu_{F}\right) \equiv \mathcal{H}^{u}\left(x, \xi, t, \mu_{F}\right)-\mathcal{H}^{d}\left(x, \xi, t, \mu_{F}\right)$, whereas the charged pion $\left(\pi^{-}\right)$production on the neutron has larger sensitivity to the negative- $x$ domain,

$$
\begin{aligned}
\mathcal{H}_{n \rightarrow \pi^{-} p}\left(x, \xi, t, \mu_{F}\right) \equiv & -\frac{2}{3} \mathcal{H}^{(3)}\left(-x, \xi, t, \mu_{F}\right) \\
& -\frac{1}{3} \mathcal{H}^{(3)}\left(x, \xi, t, \mu_{F}\right) .
\end{aligned}
$$

Since $\pi^{-}$cannot be produced on protons, we believe that the observation of this process on the liquid deuterium target will provide a clean test of GPD $\mathcal{H}^{(3)}$ at negative $x$. Similarly, measurement of $\pi^{0}$ on deuterium gives access to a combination

$$
\mathcal{H}^{u+d}\left(x, \xi, t, \mu_{F}\right)-\mathcal{H}^{u+d}\left(-x, \xi, t, \mu_{F}\right) .
$$

For processes with strangeness production, the contribution of the sea quarks is small in JLab kinematics and cancels under the assumption that the sea is flavor symmetric, for this reason they provide a relatively clean probes of the GPDs of the valence $u$ - and $d$-quarks. We expect that the process $e n \rightarrow e K^{+} \Sigma^{-}$should be relatively easy to access experimentally, since all produced hadrons are charged.

\section{B. Twist-3 corrections}

As was discussed earlier, in modern high-luminosity experiments a large part of the data come from the region of $Q$ only 2 or 3 times larger than nucleon mass $m_{N}$. For this reason, it is known that in certain channels a significant contribution comes from the higher-twist contributions due to transversely polarized photons. This evaluation is very complicated since in the same order we also have contributions of three-particle correlators which are completely unknown at present. Under the assumption that these threeparticle distributions are negligible, the result takes the form

$$
\delta \mathcal{H}_{\nu^{\prime} \lambda^{\prime}, \nu \lambda}^{q}=\left(m_{\nu^{\prime} \nu}^{q} \delta_{\lambda,-} \delta_{\lambda^{\prime},+}+n_{\nu^{\prime} \nu}^{q} \delta_{\lambda,+} \delta_{\lambda^{\prime},-}\right),
$$

where the coefficients $m_{ \pm, \pm}^{q}$ and $n_{ \pm, \pm}^{q}$ are linear combinations of the transversity GPDs,

$$
\begin{gathered}
m_{--}^{q}=\frac{\sqrt{-t^{\prime}}}{4 m}\left[2 \tilde{H}_{T}^{q}+(1+\xi) E_{T}^{q}-(1+\xi) \tilde{E}_{T}^{q}\right], \\
m_{-+}^{q}=\sqrt{1-\xi^{2}} \frac{t^{\prime}}{4 m^{2}} \tilde{H}_{T}^{q}, \\
m_{+-}^{q}=\sqrt{1-\xi^{2}}\left[H_{T}^{q}-\frac{\xi^{2}}{1-\xi^{2}} E_{T}^{q}+\frac{\xi}{1-\xi^{2}} \tilde{E}_{T}^{q}-\frac{t^{\prime}}{4 m^{2}} \tilde{H}_{T}^{q}\right], \\
m_{++}^{q}=\frac{\sqrt{-t^{\prime}}}{4 m}\left[2 \tilde{H}_{T}^{q}+(1-\xi) E_{T}^{q}+(1-\xi) \tilde{E}_{T}^{q}\right], \\
n_{--}^{q}=-\frac{\sqrt{-t^{\prime}}}{4 m}\left(2 \tilde{H}_{T}^{q}+(1-\xi) E_{T}^{q}+(1-\xi) \tilde{E}_{T}^{q}\right), \\
n_{-+}^{q}=\sqrt{1-\xi^{2}}\left(H_{T}^{q}-\frac{\xi^{2}}{1-\xi^{2}} E_{T}^{q}+\frac{\xi}{1-\xi^{2}} \tilde{E}_{T}^{q}-\frac{t^{\prime}}{4 m^{2}} \tilde{H}_{T}^{q}\right), \\
n_{++}^{q}=-\frac{\sqrt{-t^{\prime}}}{4 m}\left(2 \tilde{H}_{T}^{q}+(1+\xi) E_{T}^{q}-(1+\xi) \tilde{E}_{T}^{q}\right),
\end{gathered}
$$

and we introduced the shorthand notation $t^{\prime}=$ $-\Delta_{\perp}^{2} /\left(1-\xi^{2}\right) ; \Delta_{\perp}=p_{2, \perp}-p_{1, \perp}$ is the transverse part 
of the momentum transfer. The coefficient function (29) also gets an additional contribution nondiagonal in parton helicity,

$\delta \mathcal{C}_{\lambda^{\prime} 0, \lambda \mu}^{q}=\left(\delta_{\mu,-} \delta_{\lambda,+} \delta_{\lambda^{\prime},-}-\delta_{\mu,+} \delta_{\lambda,-} \delta_{\lambda^{\prime},+}\right) S_{V}^{q}+\mathcal{O}\left(\frac{m^{2}}{Q^{2}}\right)$,

where we introduced shorthand notations

$$
\begin{gathered}
S_{V}^{q}=\int d z\left(\left(\eta_{V+}^{q} c_{+}^{(3, p)}(x, \xi)+\eta_{V-}^{q} c_{-}^{(3, p)}(x, \xi)\right)\right. \\
\left.+2\left(\eta_{V+}^{q} c_{+}^{(3, \sigma)}(x, \xi)-\eta_{V-}^{q} c_{-}^{(3, \sigma)}(x, \xi)\right)\right), \\
c_{+}^{(3, i)}(x, \xi)=\frac{4 \pi i \alpha_{s} f_{\pi} \xi}{9 Q^{2}} \int_{0}^{1} d z \frac{\phi_{3, i}(z)}{z(x+\xi)^{2}}, \\
c_{-}^{(3, i)}(x, \xi)=\frac{4 \pi i \alpha_{s} f_{\pi} \xi}{9 Q^{2}} \int_{0}^{1} d z \frac{\phi_{3, i}(z)}{(1-z)(x-\xi)^{2}}
\end{gathered}
$$

and the twist- 3 pion distributions are defined as

$$
\begin{aligned}
\phi_{3}^{(p)}(z)= & \frac{1}{f_{\pi} \sqrt{2}} \frac{m_{u}+m_{d}}{m_{\pi}^{2}} \int \frac{d u}{2 \pi} e^{i(z-0.5) u}\langle 0| \bar{\psi}\left(-\frac{u}{2} n\right) \\
& \times \gamma_{5} \psi\left(\frac{u}{2} n\right)|\pi(q)\rangle, \\
\phi_{3}^{(\sigma)}(z)= & \frac{3 i}{\sqrt{2} f_{\pi}} \frac{m_{u}+m_{d}}{m_{\pi}^{2}} \int \frac{d u}{2 \pi} e^{i(z-0.5) u}\langle 0| \bar{\psi}\left(-\frac{u}{2} n\right) \\
& \times \sigma_{+-} \gamma_{5} \psi\left(\frac{u}{2} n\right)|\pi(q)\rangle .
\end{aligned}
$$

Thanks to the symmetry of $\phi_{p}$ and the antisymmetry of $\phi_{\sigma}$ with respect to charge conjugation, the dependence on the pion DAs factorizes in the collinear approximation and contributes only as the minus first moment of the linear combination of the twist-3 DAs, $\phi_{p}(z)+2 \phi_{\sigma}(z)$

$$
\left\langle\phi_{3}^{-1}\right\rangle=\int_{0}^{1} d z \frac{\phi_{3}^{(p)}(z)+2 \phi_{3}^{(\sigma)}(z)}{z} .
$$

In the general case the coefficient function (31) leads to collinear divergencies near the points $x= \pm \xi$ when substituted to (16). As was noted in [55], this singularity is naturally regularized by maintaining the small transverse momentum of the quarks inside the meson. Such regularization modifies (31) to

$$
\begin{aligned}
c_{+}^{(3, i)}(x, \xi)= & \frac{4 \pi i \alpha_{s} f_{\pi} \xi}{9 Q^{2}} \int_{0}^{1} d z d^{2} l_{\perp} \\
& \times \frac{\phi_{3, i}\left(z, \ell_{\perp}\right)}{(x+\xi-i 0)\left(z(x+\xi)+\frac{2 \xi \ell_{\perp}^{2}}{Q^{2}}\right)},
\end{aligned}
$$

$$
\begin{aligned}
c_{-}^{(3, i)}(x, \xi)= & \frac{4 \pi i \alpha_{s} f_{\pi} \xi}{9 Q^{2}} \int_{0}^{1} d z d^{2} l_{\perp} \\
& \times \frac{\phi_{3, i}\left(z, \ell_{\perp}\right)}{(x-\xi+i 0)\left((1-z)(x-\xi)-\frac{2 \xi \ell_{\perp}^{2}}{Q^{2}}\right)},
\end{aligned}
$$

where $\ell_{\perp}$ is the transverse momentum of the quark, and we tacitly assume absence of any other transverse momenta in the coefficient function.

\section{RESULTS AND DISCUSSION}

In this section we would like to present numerical results for charged current pion production. For the sake of definiteness, for numerical estimates we use the KrollGoloskokov parametrization of GPDs [37,38,51,55,68]. We would like to comment briefly on the inclusion of the so-called $t$-channel pion pole. As was shown long ago, this contribution can be incorporated into the GPD $\tilde{E}$ $[17,55,69]$, and gives the dominant contribution at small- $t$,

$$
\begin{aligned}
\tilde{E}_{(\mathrm{pole})}^{u}(x, \xi, t) & =-\tilde{E}_{(\mathrm{pole})}^{d}(x, \xi, t) \\
& =\theta(|x|<\xi) \Phi_{\pi}\left(\frac{x+\xi}{2 \xi}\right) \frac{F_{P}(t)}{4 \xi}, \\
F_{P}(t) & =\frac{2 \sqrt{2} m f_{\pi} g_{\pi N N}}{t-m_{\pi}^{2}} \frac{\Lambda_{N}^{2}-m_{\pi}^{2}}{\Lambda_{N}^{2}-t^{\prime}},
\end{aligned}
$$

where $t^{\prime} \equiv t-t_{\min }=-\Delta_{\perp}^{2} /\left(1-\xi^{2}\right)$, and following [55] we use constants $g_{\pi N N} \approx 13.6, \Lambda_{N} \approx 0.51 \mathrm{GeV}$. However, when we consider kaon production, we expect that the pole should be located at $t \sim m_{K}^{2}$ rather than at $t \sim m_{\pi}^{2}$. Physically, this contradiction signals that the $S U(3)$-based symmetry relations, which relate the transition GPDs $N \rightarrow Y$ to GPDs of the proton, might require modification near $|t| \lesssim m_{K}^{2}$. In what follows, we make proper adjustments of the pole term in transition GPDs, e.g., for $K^{+}$production we use $[27,69]$

$$
\begin{aligned}
\tilde{E}_{(\text {pole })}^{u}(x, \xi, t) & =-\tilde{E}_{(\text {pole })}^{s}(x, \xi, t) \\
= & \theta(|x|<\xi) \Phi_{K}\left(\frac{x+\xi}{2 \xi}\right) \frac{F_{P, K}(t)}{4 \xi}, \\
F_{P, K}(t) & =\frac{2 \sqrt{2} m f_{K} g_{K N Y}}{t-m_{K}^{2}} \frac{\Lambda_{N}^{2}-m_{K}^{2}}{\Lambda_{N}^{2}-t^{\prime}} .
\end{aligned}
$$

For numerical estimates, we assume that $g_{K N Y} \approx g_{\pi N N}$. We expect that the kaon pole contribution should be more suppressed than the pion pole contribution. For both the pion and kaon leading-twist wave function we use the asymptotic form, $\phi_{2}(z)=6 z(1-z)$, and due to the symmetry $z \rightarrow$ $1-z$ the contributions (37) and (39) are even functions of the variable $x$. For estimates of the twist-3 contribution introduced in Sec. II, we use the parametrization suggested in $[51,55]$, 

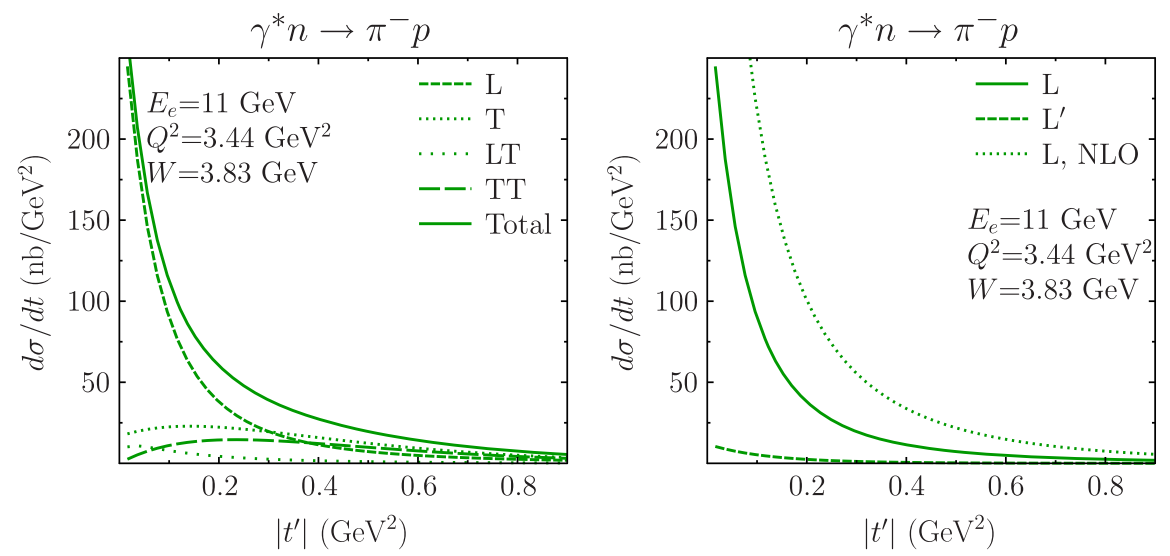

FIG. 2. Left: Different components of charged pion production on a neutron, $e n \rightarrow e \pi^{-} p$. The dominant contribution to the total cross section (solid line) comes from the longitudinal cross section $d \sigma_{L} / d t$ due to the $t$-channel pion pole. At larger $t$ the contributions of transversity GPDs become comparable to the leading-twist contribution $d \sigma_{L} / d t$. Right: Comparison of leading twist cross sections, taking into account the pole term (solid curve, marked with symbol " $L$ ") and without it (dashed curve, marked with symbol " $L$ '"). Also, in the same plot we have shown the NLO corrections to the coefficient functions. For the ease of comparison, in both plots we have chosen the same values of $W, Q^{2}$ as in [55]. The value of Bjorken $x_{B}$ is $x_{B} \approx 0.2$.

$$
\begin{aligned}
\phi_{3}\left(z, l_{\perp}\right) & =\phi_{3 ; p}\left(z, l_{\perp}\right)+2 \phi_{3 ; \sigma}\left(z, l_{\perp}\right) \\
& =\frac{16 \pi^{3 / 2}}{\sqrt{6}} f_{\pi} a_{\pi}^{3} l_{\perp} \phi_{a s}(z) \exp \left(-a_{p}^{2} l_{\perp}^{2}\right), \\
a_{\pi} & =\left[\sqrt{8} \pi f_{\pi}\right]^{-1}, \quad a_{p} \approx 2 \mathrm{GeV}^{-1} .
\end{aligned}
$$

We would like to start our discussion with the process $e n \rightarrow e \pi^{-} p$, which is sensitive to the GPD $\mathcal{H}^{(3)}=\mathcal{H}^{(u)}-\mathcal{H}^{(d)}$. As was indicated in Sec. II A, this process probes the same GPD as in the case of the charged pion production on the proton $\left(e p \rightarrow e \pi^{+} n\right)$, but is expected to have stronger sensitivity to the region of negative $x$. In Fig. 2 we have shown different components of the cross section defined in Eq. (1). Just as in the proton case, the cross section of this process at small $t$ is dominated by the $t$-channel pion pole, and since the corresponding pole contribution to the GPD $\tilde{E}$ is symmetric with respect to $x \leftrightarrow-x$, as could be seen from Table I, its contribution to the amplitudes of $e p \rightarrow e \pi^{+} n$ and $e n \rightarrow$ $e \pi^{-} p$ processes differs only by sign. At larger values of $t$, the pion pole contribution fades out, the contribution of the transverse cross section becomes more pronounced and eventually becomes comparable to $d \sigma_{L} / d t$. In the right panel of Fig. 2 we compare the values of the cross section $d \sigma_{L} / d t$ with and without pole contributions (solid and dashed lines respectively) and demonstrate the importance of the pion pole contribution. Also we have shown with a dashed line the result when the NLO corrections to the coefficient functions are taken into account. As we can see, the corrections are large, and similar conclusions are valid for other processes mentioned below. For this reason we


FIG. 3. Left: Different components of neutral pion production on the neutron, $e n \rightarrow e \pi^{0} p$. The dominant contribution to the total cross section (solid line) comes from the transverse cross section $d \sigma_{T} / d t$. Other components (not shown) are negligible. Right: The same plot for the incoherent process on the deuteron, $e D \rightarrow e \pi^{0} p n$. For ease of comparison, in both plots we have chosen the same values of $W$, $Q^{2}$ as in [55]. The value of the Bjorken $x_{B}$ is $x_{B} \approx 0.2$. 

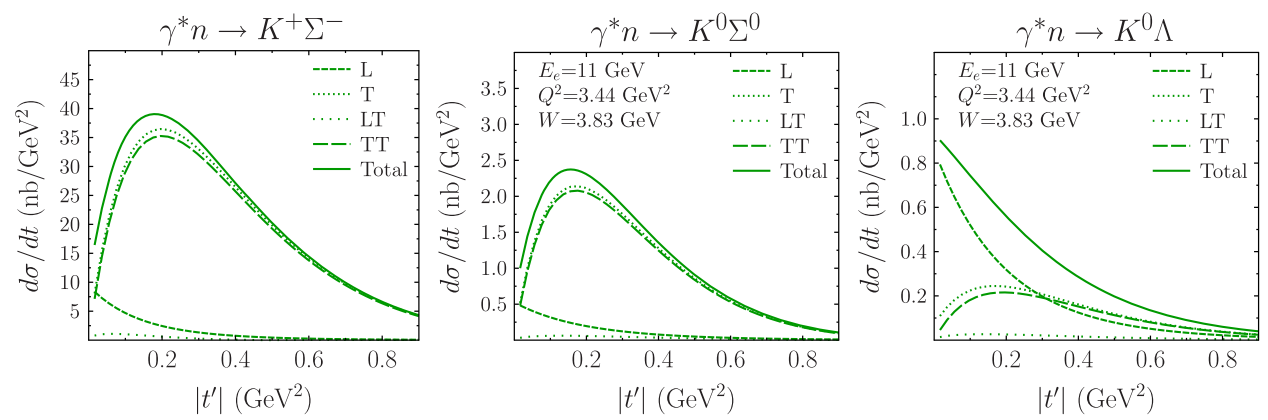

FIG. 4. Cross sections for the strangeness production on the neutron. In case of $\gamma^{*} n \rightarrow K^{+} \Sigma^{-}$and $\gamma^{*} n \rightarrow K^{0} \Sigma^{0}$ the dominant contribution to the total cross section (solid line) comes from the transverse cross section $d \sigma_{T} / d t$. In the process $\gamma^{*} n \rightarrow K^{0} \Lambda$ the dominant contribution comes from $d \sigma_{L} / d t$ (dashed line) which is mildly enhanced at small- $t^{\prime}$ due to kaon pole (39). For the ease of comparison, in both plots we have chosen the same values of $W, Q^{2}$ as in [55]. The value of the Bjorken $x_{B}$ is $x_{B} \approx 0.2$.

believe that the GPD analysis from data done in [51,55] should be repeated taking into account NLO corrections. We expect that the process $e n \rightarrow e \pi^{-} p$ could be relatively easy to access experimentally on the deuterium target, since all final hadrons are charged, and $\pi^{-}$can be produced only on protons. It is also very interesting to measure the ratio of the cross sections $d \sigma_{L} / d t$ in $e p \rightarrow e \pi^{+} n$ and $e n \rightarrow e \pi^{-} p$ processes: in the case of pion pole dominance it is expected that this ratio should be close to unity.

In Fig. 3 we show the cross sections for the case of neutral pions. The contribution of the pion pole (37) in the amplitude exactly cancels in this case due to the symmetry of (37) with respect to $x \leftrightarrow-x$ and the structure of the flavor factors (see Table I). For this reason the cross section $d \sigma_{L} / d t$ is significantly smaller, and the cross section is dominated by the transverse terms. The neutral pion production $\left(e n \rightarrow e \pi^{0} n\right)$ has the same final state as in the proton case and is experimentally indistinguishable from it. Experimentally, the $\pi^{0}$ production on neutrons might be measured either in coherent processes $e A \rightarrow e A \pi^{0}$ or in incoherent processes $e A \rightarrow e n X$. The contributions of the coherent process peak at small- $t$ and significantly depend on the implemented model of nuclear structure [70]. In contrast, the contribution of the incoherent process has a milder dependence on nuclear effects and is not suppressed at moderate $t$. The cleanest process for such a study is the production on a deuterium target, $e D \rightarrow e \pi^{0} p n$, which has negligibly small nuclear effects due to very weak binding of deuteron. The feasibility of such measurements has been demonstrated recently at Hall A $[45,48]$, although due to the low energy of the incident electron beam the virtuality $Q^{2}$ was too low for its consideration in the Bjorken limit. The cross section for the process $e D \rightarrow e \pi^{0} p n$ is shown for the sake of reference in the right panel of Fig. 3 . As could be seen from the plot, it gets the dominant contribution from the transversity GPDs.

Finally, in Fig. 4 we show predictions for the differential cross section of kaon production $\left(K^{+}\right.$and $\left.K^{0}\right)$. The sea quark densities are small in the kinematics of JLab, and additionally, as could be seen from the structure of the flavor factors in Table I, the contribution of the sea quarks cancels in the case of the flavor symmetric sea, as implemented in the parametrization [51,55], and thus kaon production is sensitive to the valence $u$ - and $d$-quarks. The largest cross section has the process en $\rightarrow e K^{+} \Sigma^{-}$, which gets the dominant contribution from the transversity GPDs of the $u$-quarks. We expect that experimentally it should be very easy to access it since both produced hadrons are charged, and additionally there is no interference from protons. The process $e p \rightarrow$ $e^{\prime} K^{0} \Sigma^{0}$ is sensitive to the same valence $u$-quarks, however, as can be seen from the flavor factors in Table I, it is suppressed compared to $K^{+} \Sigma^{-}$by a relative factor $\sim\left(e_{d} / e_{u}\right)^{2} / 2$ which results in approximately an order of magnitude smaller cross sections. Finally, the process $e n \rightarrow e K^{0} \Lambda$ has the smallest cross section due to the suppression by a relative factor $\sim\left(e_{d} / e_{u}\right)^{2} / 6$ and additionally is suppressed due to a very specific combination of GPDs $2 \mathcal{H}^{d}-\mathcal{H}^{u}$ which contribute to it, so we believe that it will be challenging to access it experimentally.

\section{CONCLUSIONS}

In this paper we analyzed the production of pions and kaons on neutron targets using the modern GPD parametrization $[37,38,51,55,68]$. We estimated the cross sections in the kinematics of upgraded $12 \mathrm{GeV}$ Jefferson Laboratory experiments, assuming that the measurements will be done on liquid deuterium targets. We found that four processes $\left(\gamma^{*} n \rightarrow \pi^{-} p, K^{+} \Sigma^{-}, K^{0} \Sigma^{+}, K^{0} \Lambda\right)$ might proceed only on neutrons and thus provide clean probes of the corresponding GPDs combinations. They do not get contributions from gluons nor from sea quarks (provided the sea is flavor symmetric), and thus probe valence GPDs of $u$ - and $d$-quarks. The neutral pion production $\left(\gamma^{*} N \rightarrow \pi^{0} N\right)$ gets comparable contributions from both proton and neutrons and thus is more challenging for the extraction of GPDs. The cross sections of pion and charged kaon production are sufficiently large and comparable to the corresponding processes on the protons. Additionally, in 
the case of charged pion and kaon production all produced hadrons are charged, which facilitates the reconstruction of the kinematics of the process and allows measurements with reasonable statistics. The code for evaluation of the cross sections with arbitrary GPD models is available from the authors on demand.

\section{ACKNOWLEDGMENTS}

This research was partially supported by Proyecto Basal FB 0821 (Chile), the Fondecyt (Chile) Grants No. 1180232 and No. 1140377, CONICYT (Chile) Grant No. PIA
ACT1413. Powered@NLHPC: This research was partially supported by the supercomputing infrastructure of the National Laboratory for High Performance Computing (NLHPC) (ECM-02). Also, we thank Yuri Ivanov for technical support of the USM HPC cluster where part of the evaluations were done.

\section{APPENDIX: NLO COEFFICIENT FUNCTION}

The function $T^{(1)}(v, z)$ in (15) encodes NLO corrections to the coefficient function.

Explicitly, this function is given by

$$
\begin{aligned}
T^{(1)}(v, z)= & \frac{1}{2 v z}\left[\frac{4}{3}\left([3+\ln (v z)] \ln \left(\frac{Q^{2}}{\mu_{F}^{2}}\right)+\frac{1}{2} \ln ^{2}(v z)+3 \ln (v z)-\frac{\ln \bar{v}}{2 \bar{v}}-\frac{\ln \bar{z}}{2 \bar{z}}-\frac{14}{3}\right)+\beta_{0}\left(\frac{5}{3}-\ln (v z)-\ln \left(\frac{Q^{2}}{\mu_{R}^{2}}\right)\right)\right. \\
& -\frac{1}{6}\left(2 \frac{\bar{v} v^{2}+\bar{z} z^{2}}{(v-z)^{3}}\left[\mathrm{Li}_{2}(\bar{z})-\mathrm{Li}_{2}(\bar{v})+\mathrm{Li}_{2}(v)-\mathrm{Li}_{2}(z)+\ln \bar{v} \ln z-\ln \bar{z} \ln v\right]\right. \\
& +2 \frac{v+z-2 v z}{(v-z)^{2}} \ln (\bar{v} \bar{z})+2\left[\mathrm{Li}_{2}(\bar{z})+\mathrm{Li}_{2}(\bar{v})-\mathrm{Li}_{2}(z)-\mathrm{Li}_{2}(v)+\ln \bar{v} \ln z+\ln \bar{z} \ln v\right] \\
& \left.\left.+4 \frac{v z \ln (v z)}{(v-z)^{2}}-4 \ln \bar{v} \ln \bar{z}-\frac{20}{3}\right)\right],
\end{aligned}
$$

where $\beta_{0}=\frac{11}{3} N_{c}-\frac{2}{3} N_{f}, \mathrm{Li}_{2}(z)$ is the dilogarithm function, and $\mu_{R}$ and $\mu_{F}$ are the renormalization and factorization scales respectively. For the vector meson production in processes when the internal state of the hadron is not changed, the additional contribution comes from gluons and singlet (sea) quarks $[33,64,65]{ }^{1}$

Some coefficient functions have nonanalytic behavior $\sim \ln ^{2} v$ for small $v \approx 0(x= \pm \xi \mp i 0)$, which signals that the collinear approximation might be not valid near this point. This singularity in the collinear limit occurs due to the omission of the small transverse momentum $l_{M, \perp}$ of

${ }^{1}$ For the sake of simplicity, we follow [33] and assume that the factorization scale $\mu_{F}$ is the same for both the generalized parton distribution and the pion distribution amplitude. the quark inside a meson [55]. For this reason the contribution of the region $|v| \sim l_{M, \perp}^{2} / Q^{2}$ for finite $Q^{2}$ (below the Bjorken limit) should be treated with due care. However, a full evaluation of $T^{(1)}(v, z)$ beyond the collinear approximation (taking into account all higher twist corrections) presents a challenging problem and has not been done so far. It was observed in [33], that the singular terms might be eliminated by a redefinition of the renormalization scale $\mu_{R}$, however near the point $v \approx 0$ the scale $\mu_{R}^{2}$ becomes soft, $\mu_{R}^{2} \sim z v Q^{2} \lesssim l_{\perp}^{2}$, which is another manifestation that nonperturbative effects become relevant. For this reason, sufficiently large value of $Q^{2}$ should be used to mitigate contributions of higher twist effects.
[1] X. D. Ji and J. Osborne, Phys. Rev. D 58, 094018 (1998).

[2] J. C. Collins and A. Freund, Phys. Rev. D 59, 074009 (1999).

[3] M. Diehl, Phys. Rep. 388, 41 (2003).

[4] J. Koempel, P. Kroll, A. Metz, and J. Zhou, Phys. Rev. D 85, 051502 (2012).

[5] G. Duplančić, K. Passek-Kumerički, B. Pire, L. Szymanowski, and S. Wallon, arXiv:1809.08104.
[6] B. Pire and L. Szymanowski, Phys. Rev. D 96, 114008 (2017).

[7] A. Pedrak, B. Pire, L. Szymanowski, and J. Wagner, Phys. Rev. D 96, 074008 (2017).

[8] R. Boussarie, B. Pire, L. Szymanowski, and S. Wallon, arXiv:1708.09164.

[9] R. Boussarie, B. Pire, L. Szymanowski, and S. Wallon, J. High Energy Phys. 02 (2017) 054. 
[10] J. P. Lansberg, L. Szymanowski, and J. Wagner, J. High Energy Phys. 09 (2015) 087.

[11] D. Y. Ivanov, A. Schafer, L. Szymanowski, and G. Krasnikov, Eur. Phys. J. C 34, 297 (2004); 75, 75(E) (2015).

[12] R. Dupré, M. Guidal, S. Niccolai, and M. Vanderhaeghen, arXiv:1704.07330.

[13] N. H. Saylor et al., arXiv:1810.02110.

[14] M. Diehl and S. Sapeta, Eur. Phys. J. C 41, 515 (2005).

[15] A. V. Belitsky and D. Mueller, Phys. Lett. B 417, 129 (1998).

[16] X. D. Ji, Phys. Rev. D 55, 7114 (1997).

[17] M. Vanderhaeghen, P. A. M. Guichon, and M. Guidal, Phys. Rev. D 60, 094017 (1999).

[18] M. Vanderhaeghen, P. A. M. Guichon, and M. Guidal, Phys. Rev. Lett. 80, 5064 (1998).

[19] C. M. Camacho et al. (Jefferson Lab Hall A and Hall A DVCS Collaborations), Phys. Rev. Lett. 97, 262002 (2006).

[20] D. Mueller, D. Robaschik, B. Geyer, F. M. Dittes, and J. Horejsi, Fortschr. Phys. 42, 101 (1994).

[21] X. D. Ji, J. Phys. G 24, 1181 (1998).

[22] A. V. Radyushkin, Phys. Lett. B 380, 417 (1996).

[23] A. V. Radyushkin, Phys. Rev. D 56, 5524 (1997).

[24] A. V. Radyushkin, arXiv:hep-ph/0101225.

[25] J. C. Collins, L. Frankfurt, and M. Strikman, Phys. Rev. D 56, 2982 (1997).

[26] S. J. Brodsky, L. Frankfurt, J. F. Gunion, A. H. Mueller, and M. Strikman, Phys. Rev. D 50, 3134 (1994).

[27] K. Goeke, M. V. Polyakov, and M. Vanderhaeghen, Prog. Part. Nucl. Phys. 47, 401 (2001).

[28] M. Diehl, T. Feldmann, R. Jakob, and P. Kroll, Nucl. Phys. B596, 33 (2001); B605, 647(E) (2001).

[29] A. V. Belitsky, D. Mueller, and A. Kirchner, Nucl. Phys. B629, 323 (2002).

[30] A. V. Belitsky and A. V. Radyushkin, Phys. Rep. 418, 1 (2005).

[31] V. Kubarovsky (CLAS Collaboration), Nucl. Phys. B, Proc. Suppl. 219-220, 118 (2011).

[32] I. V. Anikin, D. Y. Ivanov, B. Pire, L. Szymanowski, and S. Wallon, Nucl. Phys. B828, 1 (2010).

[33] M. Diehl and W. Kugler, Eur. Phys. J. C 52, 933 (2007).

[34] L. Mankiewicz, G. Piller, and A. Radyushkin, Eur. Phys. J. C 10, 307 (1999).

[35] L. Mankiewicz and G. Piller, Phys. Rev. D 61, 074013 (2000).

[36] S. Ahmad, G. R. Goldstein, and S. Liuti, Phys. Rev. D 79, 054014 (2009).

[37] S. V. Goloskokov and P. Kroll, Eur. Phys. J. C 50, 829 (2007).

[38] S. V. Goloskokov and P. Kroll, Eur. Phys. J. C 53, 367 (2008).

[39] B. Z. Kopeliovich, I. Schmidt, and M. Siddikov, Phys. Rev. D 86, 113018 (2012).

[40] B. Z. Kopeliovich, I. Schmidt, and M. Siddikov, Phys. Rev. D 87, 033008 (2013).
[41] B. Z. Kopeliovich, I. Schmidt, and M. Siddikov, Phys. Rev. D 89, 053001 (2014).

[42] M. Siddikov and I. Schmidt, Phys. Rev. D 95, 013004 (2017).

[43] I. Schmidt and M. Siddikov, Phys. Rev. D 91, 073002 (2015).

[44] M. Mazouz et al. (Jefferson Lab Hall A Collaboration), Phys. Rev. Lett. 99, 242501 (2007).

[45] M. Mazouz et al. (Jefferson Lab Hall A Collaboration), Phys. Rev. Lett. 118, 222002 (2017).

[46] D. H. Ho et al. (CLAS Collaboration), arXiv:1805.04561.

[47] N. Compton et al. (CLAS Collaboration), Phys. Rev. C 96, 065201 (2017).

[48] P. E. Bosted et al. (CLAS Collaboration), Phys. Rev. C 94, 055201 (2016).

[49] M. Defurne et al. (Jefferson Lab Hall A Collaboration), Phys. Rev. Lett. 117, 262001 (2016).

[50] G. R. Goldstein, J. O. G. Hernandez, and S. Liuti, arXiv:1311.0483.

[51] S. V. Goloskokov and P. Kroll, Eur. Phys. J. A 47, 112 (2011).

[52] T. Horn, Phys. Rev. C 85, 018202 (2012).

[53] T. Horn, Proc. Sci. DIS2017 (2018) 115.

[54] M. Carmignotto et al., Phys. Rev. C 97, 025204 (2018).

[55] S. V. Goloskokov and P. Kroll, Eur. Phys. J. C 65, 137 (2010).

[56] L. L. Frankfurt, P. V. Pobylitsa, M. V. Polyakov, and M. Strikman, Phys. Rev. D 60, 014010 (1999).

[57] T. Arens, O. Nachtmann, M. Diehl, and P. V. Landshoff, Z. Phys. C 74, 651 (1997).

[58] X. Zheng et al. (Jefferson Lab Hall A Collaboration), Phys. Rev. C 70, 065207 (2004).

[59] J. Huang et al. (Jefferson Lab Hall A Collaboration), Phys. Rev. Lett. 108, 052001 (2012).

[60] D. Flay et al. (Jefferson Lab Hall A Collaboration), Phys. Rev. D 94, 052003 (2016).

[61] A. Airapetian et al. (HERMES Collaboration), arXiv: 1810.07054.

[62] O. A. Rondon-Aramayo, Phys. Rev. C 60, 035201 (1999).

[63] B. Z. Kopeliovich, I. Schmidt, and M. Siddikov, Nucl. Phys. A918, 41 (2013).

[64] A. V. Belitsky and D. Mueller, Phys. Lett. B 513, 349 (2001).

[65] D. Y. Ivanov, L. Szymanowski, and G. Krasnikov, Pis'ma Zh. Eksp. Teor. Fiz. 80, 255 (2004) [JETP Lett. 80, 226 (2004)]; 101, 844(E) (2015).

[66] E. Braaten and S. M. Tse, Phys. Rev. D 35, 2255 (1987).

[67] B. Melic, B. Nizic, and K. Passek, Phys. Rev. 60, 074004 (1999).

[68] S. V. Goloskokov and P. Kroll, Eur. Phys. J. C 59, 809 (2009).

[69] M. Penttinen, M. V. Polyakov, and K. Goeke, Phys. Rev. D 62, 014024 (2000).

[70] V. Guzey and M. Siddikov, J. Phys. G 32, 251 (2006). 